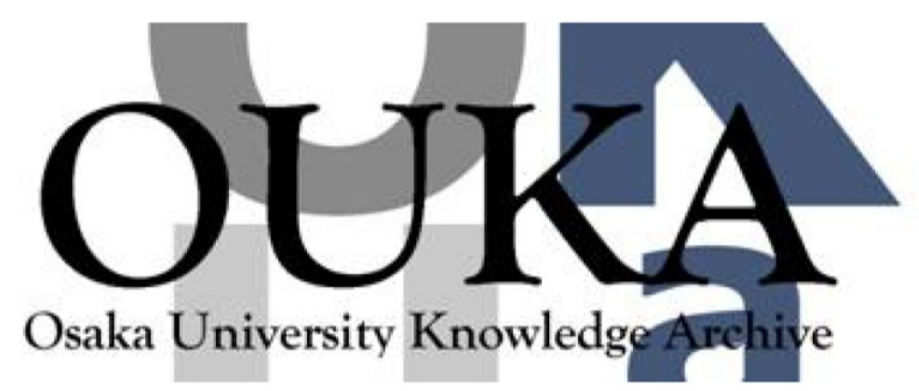

\begin{tabular}{|c|c|}
\hline Title & $\begin{array}{l}\text { London Penetration Depth of Heavy-Fermion } \\
\text { Superconductors }\end{array}$ \\
\hline Author (s) & Varma, C. M.; Miyake, K.; Schmitt-Rink, S. \\
\hline Citation & Physical Review Letters. 57(5) p. 626-p. 628 \\
\hline Issue Date & $1986-08-04$ \\
\hline oaire:version & VoR \\
\hline URL & https://hdl. handle. net/11094/3163 \\
\hline rights & $\begin{array}{l}\text { Varma, C. M., Miyake, K., Schmitt-Rink, S., } \\
\text { Physical Review Letters, 57, 5, 626-628, 1986- } \\
\text { 08-04. "Copyright } 1986 \text { by the American Physical } \\
\text { Society." }\end{array}$ \\
\hline Note & \\
\hline
\end{tabular}

Osaka University Knowledge Archive : OUKA

https://ir. Library. osaka-u. ac. jp/

0saka University 


\title{
London Penetration Depth of Heavy-Fermion Superconductors
}

\author{
C. M. Varma, K. Miyake, ${ }^{(a)}$ and S. Schmitt-Rink \\ AT\&T Bell Laboratories, Murray Hill, New Jersey 07974 \\ (Received 9 May 1986)
}

\begin{abstract}
The dynamic effective mass in heavy-fermion systems is different from the bare mass, unlike in one-component Fermi liquids. This is used to explain the appearance of a heavy mass in the London penetration depth at all temperatures. $F_{0}^{s} \sim 10^{2}$ and $F_{1}^{s} \sim 1$ are found, reflecting the strong frequency dependence but weak momentum dependence of the conduction-electron self-energy.
\end{abstract}

PACS numbers: $74.30 . \mathrm{Ci}, 72.10 . \mathrm{Bg}, 74.20 . \mathrm{Fg}$

The London penetration depth $\lambda(T)$ of the heavyfermion superconductor $\mathrm{UBe}_{13}$ has been measured recently by Einzel et al. ${ }^{1}$ This measurement probes the nature of the renormalization in a very decisive way. $\mathrm{UBe}_{13}$ has a mass enhancement $\mathrm{m}^{*} / \mathrm{m}$ of $-10^{2},{ }^{2}$ as deduced from the approximately linear specific heat in the normal state. Here, $m$ is the bare electron mass. The theory of Leggett $^{3}$ for ordinary Fermi liquids predicts that $\lambda(T)$ is renormalized from its value $\lambda_{\mathrm{BCS}}(T)$ in the BCS theory by the effective mass for $T \rightarrow T_{c}$, but has the BCS value for $T \rightarrow 0$ when there are no thermally excited quasiparticles:

$$
\begin{aligned}
& \lambda^{-2}(T)=\left(m / m^{*}\right) \lambda_{\mathrm{BCS}}^{-2}(T), \quad T \rightarrow T_{c}, \\
& \lambda^{2}(T)=\lambda_{\mathrm{BCS}}^{-2}(T), \quad T \rightarrow 0 .
\end{aligned}
$$

Here, the effects of band structure have been neglected (for which Leggett has also provided a theory). Note that $\lambda_{\mathrm{BCS}}^{2}$ is proportional to $m$.

The dramatic effect predicted by Eq. (1) is not evident in the experiments ${ }^{1}$; the heavy mass appears in $\lambda(T)$ at all temperatures. Either all the mass enhancement in heavy-fermion systems is a one-electron effect, a notion which can be rejected on other grounds, or a theory of the renormalization in heavy-fermion systems different from usual Fermi-liquid theory is required. Such a theory has been advanced recently ${ }^{4}$ to account for the contrasting renormalization seen in the static and some of the transport experiments. Here we show that this theory provides an explanation of the London penetration-depth experiments as well.

The point of view is that in Kondo lattice systems the local moments are confined for low-temperature and low-frequency experiments. Their entropy appears in a fermionic form renormalizing the conduction-electron mass for $T<T_{S}$, which is the characteristic confinement temperature, related to the Kondo temperature as modified by the presence of a dense set of magnetic ions. If $T_{S} \ll E_{\mathrm{F}}$, the conductionelectron Fermi energy, and the momentum scale of the magnetic fluctuations is similar to $k_{\mathrm{F}}$, the conduction-electron self-energy $\Sigma(k, \omega)$ has the property that

$$
\partial \Sigma(k, \omega) / \partial \omega=O\left(\Sigma(k, \omega) / T_{S}\right),
$$

while

$$
\left(1 / v_{\mathrm{F}}\right) \partial \Sigma(k, \omega) / \partial k=O\left(\Sigma(k, \omega) / E_{\mathrm{F}}\right),
$$

so that the latter is negligible compared to the former. 5,6

If the $k$ dependence of $\Sigma$ is completely neglected, the compressibility

$$
\frac{d n}{d \mu}=\frac{m^{*} / m}{1+F_{0}^{s}}\left(\frac{d n}{d \mu}\right)_{0}
$$

is unrenormalized from its value for noninteracting electrons $(d n / d \mu)_{0}$. Correspondingly

$$
m^{*}=m\left(1+F_{0}^{s}\right)=m_{d}
$$

where $m_{d}$ is the dynamic effective mass

$$
m_{d} / m=[1-\partial \Sigma(k, \omega) / \partial \omega]_{k=k_{\mathrm{F}}, \omega=E_{\mathrm{F}}}
$$

and not $m^{*}=m\left(1+F_{1}^{s} / 3\right)$, as in the usual Fermiliquid theory. If the correction due to the $k$ dependence of $\Sigma$ is included, the effective mass is

$$
m^{*}=m_{d}\left(1+F_{1}^{s} / 3\right) \text {. }
$$

From (2) we expect that $F_{1}^{s} / F_{0}^{s}=O\left(T_{S} / E_{\mathrm{F}}\right)<<1$.

As has been pointed out by Leggett, ${ }^{7}$ the dynamic effective mass $m_{d}$ is just the ratio of the momentum of a quasiparticle and the current carried by it. In an ordinary one-component translationally invariant (Galilean invariant) Fermi liquid, the total current operator commutes with the Hamiltonian and therefore is the same with or without interaction. Then $m_{d}=m$. This is in general not true for a mutually interacting two-component Fermi liquid (even if translationally invariant), if the current is defined in terms of one component only. For a situation in which $\partial \Sigma / \partial k=0$, the dynamic effective mass may then simply be calculated as follows: The current operator $j$ is given by

$$
\mathbf{j}=\mathbf{p} / \mathrm{m} \text {, }
$$

where $\mathbf{p}$ is the momentum operator. Suppose that one takes matrix elements of this equation with quasiparti- 
cle wave functions $|\mathbf{k}\rangle$ :

$$
\begin{aligned}
\left\langle\mathbf{k}|\mathbf{j}| \mathbf{k}^{\prime}\right\rangle & =\left\langle\mathbf{k}|\mathbf{p}| \mathbf{k}^{\prime}\right\rangle / m \\
& =(z / m)_{0}\left\langle\mathbf{k}|\mathbf{p}| \mathbf{k}^{\prime}\right\rangle_{0},
\end{aligned}
$$

where $z$ is the wave-function renormalization defined through

$$
|\mathbf{k}\rangle=z^{1 / 2}|\mathbf{k}\rangle_{0}
$$

and $|\mathbf{k}\rangle_{0}$ are the wave functions for the corresponding noninteracting system.

For the usual one-component Fermi liquid, the second step in (8) is inconsistent with the continuity equation and therefore incorrect. It violates Galilean invariance. The mistake is that one must take into account the "backflow" of other particles about a moving particle and calculate a corrected matrix element (vertex correction). The correction term proportional to $d \Sigma / d k$ (Nozières ${ }^{8}$ ) eliminates the factor $z$ in $(8 \mathrm{~b})$.

The situation is different for heavy fermions, which are two-component objects-the conduction electrons and the local moments-only one of which, the former, carry current, and for this component $d \Sigma / d k$ is negligible. In that case, the continuity equation is satisfied by (8). ${ }^{9}$ The current is then inversely proportional to the dynamic effective mass, since

$$
m_{d} / m=z^{-1} \text {. }
$$

This means that the effective Hamiltonian for the coupling of the heavy fermions to a vector potential $\mathbf{A}$ is

$$
H_{C}=-(e / c) \mathbf{J} \cdot \mathbf{A}=-\left(e / m_{d} c\right) \mathbf{P} \cdot \mathbf{A}
$$

and not $-e \mathbf{P} \cdot \mathbf{A} / m c$ as for the one-component Fermi liquid. Here $\mathbf{P}$ is the total quasiparticle momentum operator. From gauge invariance it follows that the diamagnetic energy term for heavy fermions is

$$
H_{M}=e^{2} A^{2} / 2 m_{d} c^{2} \text {. }
$$

The lack of Galilean invariance in this problem has nothing to do with lattice periodicity. Instead there is some similarity with the motion of a ${ }^{3} \mathrm{He}$ atom in liquid ${ }^{4} \mathrm{He}$, where the ${ }^{3} \mathrm{He}$ current depends on a dynamic effective mass $m^{*} /\left(1+F_{1}^{s} / 3\right)>m$, because unmoving ${ }^{4} \mathrm{He}$ provides a fixed frame of reference.

We can now use (11) and (12) and Leggett's phenomenological approach to derive the London penetration depth. The Hamiltonian is expressed as in Ref. 3:

$$
H=H_{\mathrm{QP}}+H_{L}+H_{C}+H_{M},
$$

where $H_{\mathrm{QP}}$ is the BCS Hamiltonian written in terms of quasiparticle operators and $H_{L}$ gives rise to "molecular fields." $H_{C}$ and $H_{M}$ are given by (11) and (12), and

$$
H_{L}=[2 n(0)]^{-1} F_{1}^{s} k_{\mathrm{F}}^{-2} P^{2} \text {, }
$$

where

$$
\mathbf{P}=\sum_{\mathbf{k}, \boldsymbol{\sigma}} \mathbf{k} \delta n(\mathbf{k}, \boldsymbol{\sigma})
$$

and $n(0)$ is the density of states at the Fermi level. The Landau coefficient $F_{1}^{s}$ enters in (14) just as in Leggett's paper because $\mathbf{P}$ has dipolar symmetry. Corrections due to $F_{l}^{s}, l>1$, can be neglected, since because of (2)

$$
F_{1}^{s}=F_{0}^{s} O\left(T_{S} / E_{\mathrm{F}}\right)
$$

and higher $F_{l}^{s}$ will be smaller still. Since our concern here is primarily with renormalization effects, we will first obtain the penetration depth from (11)-(15) for isotropic superconducting states. We will discuss the anisotropic superconducting case subsequently.

For the isotropic case, the transverse gauge is sufficient to guarantee that there is no longitudinal response from the quasiparticles for an applied dc field. Then the London penetration depth is given by

$$
\lambda^{-2}(T)=\left(4 \pi e^{2} / c^{2}\right)\left[N / m_{d}-K(T)\right] .
$$

In (17) the first term is the diamagnetic contribution, $N$ is the electron density, and $m_{d}$ rather than $m$ enters, as in Leggett's case, because (12) involves $m_{d}$. The second term is the paramagnetic response

$$
K(T)=c\langle\mathbf{J}\rangle / e \mathbf{A} .
$$

The result for $\lambda$ is

$$
\lambda^{-2}(T)=\frac{4 \pi N e^{2}}{m_{d} c^{2}}\left(1-\frac{\left(1+F_{1}^{s} / 3\right) Y(T)}{1+\left(F_{1}^{s} / 3\right) Y(T)}\right),
$$

where $Y(T)$ is the Yosida function

$$
Y(T)=-[n(0)]^{-1} \Sigma_{\mathbf{k}} d n / d E_{k} .
$$

Equation (19) has the property

$$
\begin{array}{ll}
\lambda^{-2}(T)=\left(m / m^{*}\right) \lambda_{\mathrm{BCS}}^{-2}(T), & T \rightarrow T_{c}, \\
\lambda^{-2}(T)=\left(m / m_{d}\right) \lambda_{\mathrm{BCS}}^{-2}(T), & T \rightarrow 0,
\end{array}
$$

where

$$
m^{*}=m_{d}\left(1+F_{1}^{s} / 3\right)=O\left(m_{d}\right) .
$$

This should be contrasted with (1), the results for the usual Fermi liquid.

Now we briefly mention the modifications for an anisotropic superconducting order parameter, which have already been discussed. Since the superfluid density or the Yosida function in this case is anisotropic, $\lambda_{\mathrm{BCS}}(T)$ is in general a tensor. If we assume that the bare mass $m$ is isotropic, so are $m_{d}$ and $m^{*}$. At this stage of our knowledge of heavy fermions other assumptions are fruitless. Analysis of the experiments then proceeds via modification of (19) to the anisotropic case as already done by Einzel et al. ${ }^{1}$ The only change is the occurrence of $m_{d}$ in (19) instead of 
$m$ in their work.

The other modification due to anisotropy is that in a general geometry (mutual orientation of sample, anisotropy axis, and magnetic field), the transverse gauge does not guarantee zero longitudinal quasiperiodic current. Either a temperature-dependent gauge must be chosen or, as analyzed by Millis, ${ }^{10}$ in the transverse gauge the contribution of the phase collective mode to the current must be considered. However, in most simple geometries transverse gauge still guarantees zero longitudinal quasiparticle current. The experiment in any case is done on a polycrystalline sample and one may for the present ignore this subtlety.

The analysis of the experiment is then parallel to that by Einzel et al. ${ }^{1} m_{d} / m \approx F_{0}^{s}$ is found to be $-10^{2}$ and $F_{1}^{s} \sim 1$. This confirms the view adopted of the heavy fermions as it satisfies (2) and (16).

There is one minor difference of opinion from Einzel et al., 'which pertains not to the renormalizations (which are our concern here), but to the particular anisotropic state used to fit the data. They prefer an axial-like state, but within the uncertainty of the data and taking into account that strong coupling corrections to $\lambda_{\mathrm{BCS}}(T)$ have not been concerned, we feel that a polarlike state adequately fits the data. As analyzed elsewhere, nuclear relaxation rate, ultrasonic attenuation, and thermal conductivity all favor a polarlike state for $\mathrm{UBe}_{13}{ }^{11}$

One of us (C.M.V.) would like to thank G. E. Brown for urging him to analyze the Fermi-liquid corrections to the London penetration depth. A useful conversation with J. Sauls is also acknowledged.

(a) Permenant address: Department of Physics, Nagoya University, Chikusa-ku, Nagoya 464, Japan.

${ }^{1}$ D. Einzel, P. J. Hirschfeld, F. Gross, B. S. Chandrasekhar, K. Andres, H. R. Ott, J. Beuers, Z. Fisk, and J. L. Smith, to be published.

2See, e.g., G. R. Stewart, Rev. Mod. Phys. 56, 755 (1984).

${ }^{3}$ A. J. Leggett, Phys. Rev. 140, A1869 (1965).

${ }^{4}$ C. M. Varma, Phys. Rev. Lett. 55, 2723 (1985).

${ }^{5}$ C. M. Varma, Comments Solid State Phys. 11, 221 (1985)

${ }^{6} \mathrm{~A}$ similar behavior of the $f$-electron self-energy has been discussed microscopically in the context of the periodic Anderson model by $\mathrm{H}$. Jichu, T. Matsuura, and Y. Kuroda, Prog. Theor. Phys. 72, 366 (1984), and J. Magn. Magn. Mater. 52, 242 (1985). See also H. Fukuyama, in Proceedings of the Eighth Taniguchi Symposium on the Theory of the Valence Fluctuating State, Taniguchi, Japan, I984, edited by T. Kasuya (Springer-Verlag, New York, 1985).

${ }^{7}$ A. J. Leggett, Ann. Phys. (N.Y.) 46, 76 (1968).

${ }^{8} \mathrm{P}$. Nozières, The Theory of Interacting Fermi Systems (Benjamin, New York, 1964). $d \Sigma / d k$ must be distinguished from $\partial \Sigma / \partial k$ as explained by Nozières.

${ }^{9}$ R. E. Prange and L. P. Kadanoff, Phys. Rev. 134, A566 (1964).

${ }^{10} \mathrm{~A}$. J. Millis, to be published.

${ }^{11 S}$. Schmitt-Rink, K. Miyake, and C. M. Varma, to be published. 